\title{
ANALYSE ET CONSOLIDATION DES RESULTATS SUR LES ESTIMATIONS DE SUPERFICIE DU COUVERT FORESTIER ET DE SES CHANGEMENTS ENTRE 2000 ET 2016 EN REPUBLIQUE DU CONGO
}

\author{
Suspense Averti IFO ${ }^{1}$, Christophe SANNIER ${ }^{2}$, Gabriel JAFFRAIN ${ }^{3}$, Hervis GHOMSI ${ }^{2}$, Cherubins OUISSIKA $^{4}$, \\ Stoffenne BINSANGOU ${ }^{1}$, Lisa LOUVOUANDOU ${ }^{1}$, Leslie BOUETOU KADILAMIO ${ }^{4}$, Georges Claver BOUNDZANGA ${ }^{5}$, \\ Benoit MERTENS ${ }^{6}$, Camille PINET ${ }^{3}$ \\ 1 : Université Marien N'GOUABI, Brazzaville, Congo. averti.ifosuspens@umng.cg \\ 2 : Collecte Localisation Satellites, Villeneuve d'Ascq, France. csannier@groupcls.com \\ 3 : IGN FI, Paris, France. gjaffrain@ignfi.fr \\ 4 : CNIAF, Brazzaville, Congo. \\ 5 : Coordination Nationale REDD+ Congo \\ 6 : UMR Espace-DEV, Institut de recherche pour le développement (IRD)
}

\begin{abstract}
Résumé
Des rapports précis et cohérents sur l'évolution de la superficie forestière et des changements d'occupation du sol sont importants dans le contexte de l'accord de Paris où les pays ont l'obligation de soumettre régulièrement leurs rapports sur le bilan émission - absorption des gaz à effet de serre. Cette notification des changements peut avoir un impact direct sur les paiements par le biais de comparaisons avec les niveaux de référence (émissions) nationaux dans le cadre de la réduction des émissions dues à la déforestation et à la dégradation des forêts, notamment dans le cadre du processus REDD+ (Réduction des émissions issues de la déforestation et de la dégradation forestière). Cependant avant cela, les pays sont invités à renforcer leur système national de surveillance des forêts mais aussi de production des produits cartographiques qui respectent des règles robustes d'évaluation de l'incertitude des estimations de l'évolution de la superficie du couvert forestier prescrites par la CCNUCC (Convention-cadre des Nations Unies sur les changements climatiques). Dans cette étude, nous présentons les résultats de la précision des nombreuses cartes forestières dont le Congo dispose en utilisant l'approche assistée par modèle développée par Sannier et al, 2014, adaptée au contexte de la République du Congo. Les résultats de l'étude montrent une sous-estimation de près de $50 \%$ des pertes forestières dans la période entre 2000 et 2014 que ce soit par la méthode des estimations directes ou des estimations par régression. La comparaison des deux méthodes montre que les estimations des pertes de la couverture forestière par la méthode d'échantillonnage par point sous-estime l'estimation des pertes sur la période 2000-2014 ainsi que sur la période 2014-2016. Nous concluons qu'il faut renforcer les équipes nationales en charge de l'élaboration des cartes forestières mais aussi que le pays doit s'approprier cette méthode de Sannier et al. 2014 pour l'évaluation de la précision.
\end{abstract}

Mots-clés :

Couvert et perte du couvert forestier, télédétection, évaluation de la précision, Congo

\begin{abstract}
Accurate and consistent reporting on forest cover and land use / cover changes is crucial in the post-Paris agreement context where countries are obliged to regularly submit their reports on the greenhouse gas balance of emissions and removals. This reporting of changes can have a direct impact on payments through comparisons with national reference (emission) levels in the context of reducing emissions from deforestation and forest degradation, particularly as part of the REDD+ process (Reducing emissions from deforestation and forest degradation). However, before doing so, countries are invited to strengthen their national forest monitoring system and also to produce map products that comply with robust methods for assessing the uncertainty of forest cover change estimates as prescribed by UNFCCC (United Nations Framework Convention on Climate Change). In this study, we present the results of the accuracy of the numerous forest maps available in Congo using a new method developed by SIRS and adapted to the context of the Republic of Congo. The results of the study show an underestimation of nearly $50 \%$ of forest losses in the period between 2000 and 2014 either by the direct estimation method or by regression estimates. Comparison of the two methods shows that the estimates of forest cover losses by the sample point method underestimates the estimate of losses over the period 2000-2014 as well as over the period 2014-2016. We conclude that there is a need to strengthen the national teams in charge of forest mapping, but also that the method by Sannier et al. 2014 should be adopted for the evaluation of precision.
\end{abstract}

Keywords:

Forest cover and loss of forest cover, remote sensing, accuracy assessment, Congo

\section{Introduction}

\subsection{Contexte}

La république du Congo, pays forestier du bassin du Congo (figure 1), se caractérise par un massif forestier très dense, réparti du nord au sud sur trois grands massifs et dont la diversité globale des espèces forestières est excessivement élevée (figure 2).

Le domaine privé de l'Etat, qui fait partie du domaine forestier permanent (article 10, code forestier, 2020) est divisé (article 52 , code forestier, 2020) en unités forestières d'aménagement (UFA) concédées au secteur privé sur la base de conventions à durée bien déterminée. 
Cette forêt est majoritairement exploitée pour son bois (figure 3), mais aussi d'autres activités anthropiques telles que l'exploitation minière artisanale et l'agriculture itinérante (Tchatchou et al. 2015 Tyukavina et al. 2018). Suivant le rapport WRI Congo (WRI, 2017) les forêts aménagées attribuées s'étendent sur 13853814 ha, alors que les concessions forestières non attribuées occupent une superficie de 816109 ha. A noter que $13 \%$ du territoire national ont été affectés à la création des aires protégées. Les différentes activités anthropiques sur les forêts congolaises occasionnent des pertes forestières comprenant la dégradation et la déforestation, variant entre 11000 ha et 30000 ha par an (CNIAF' 2015). Selon Tyukavina et al (2018),

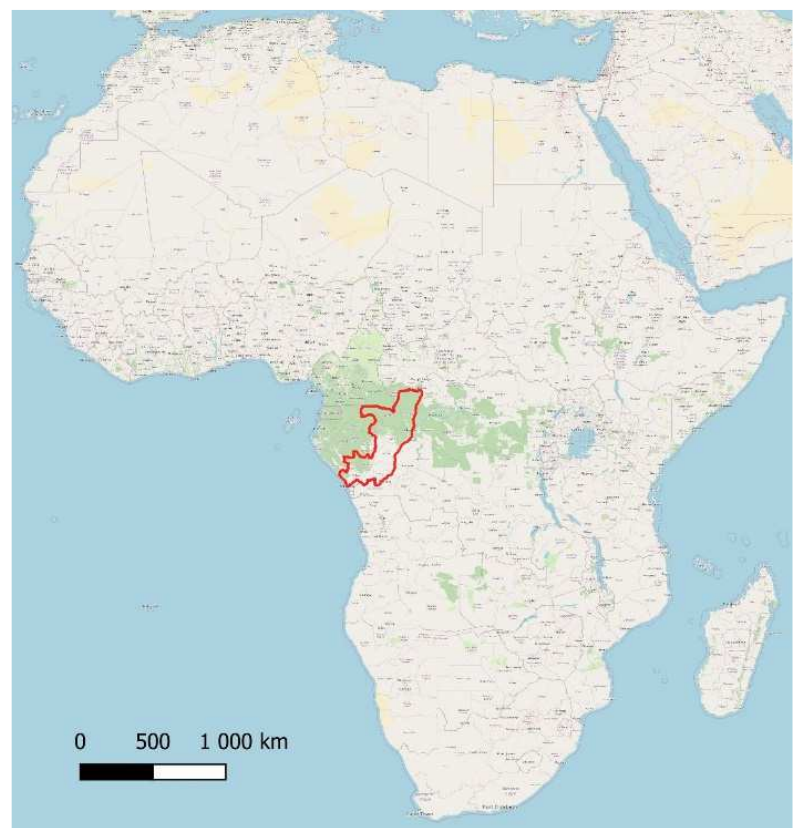

Figure 1 : Carte de localisation de la République du Congo (en rouge), en Afrique Centrale.

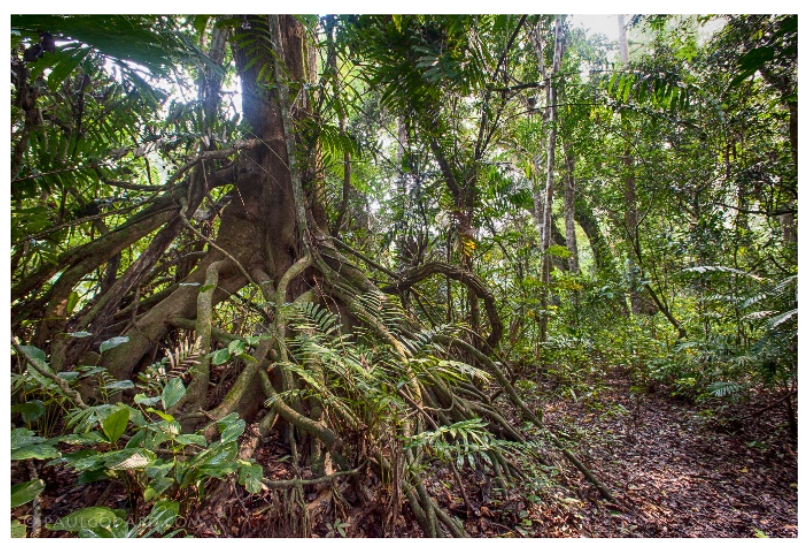

Figure 2a : Diversité végétale. Congo, département de la Cuvette-Ouest (photo : Paul Godard).

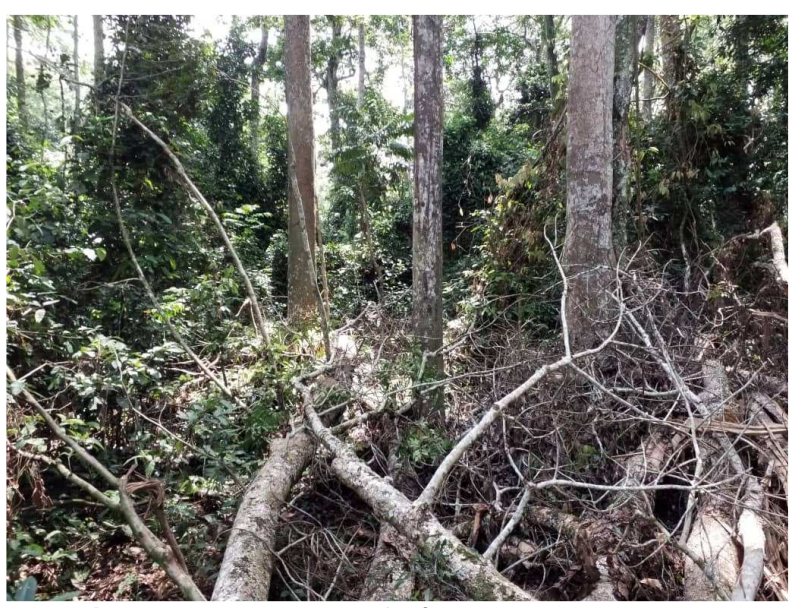

Figure $\mathbf{2 b}$ : Vue d'une forêt adulte au nord de la République du Congo dans l'UFA Pokola. (Photo : Suspense Ifo, 2019).

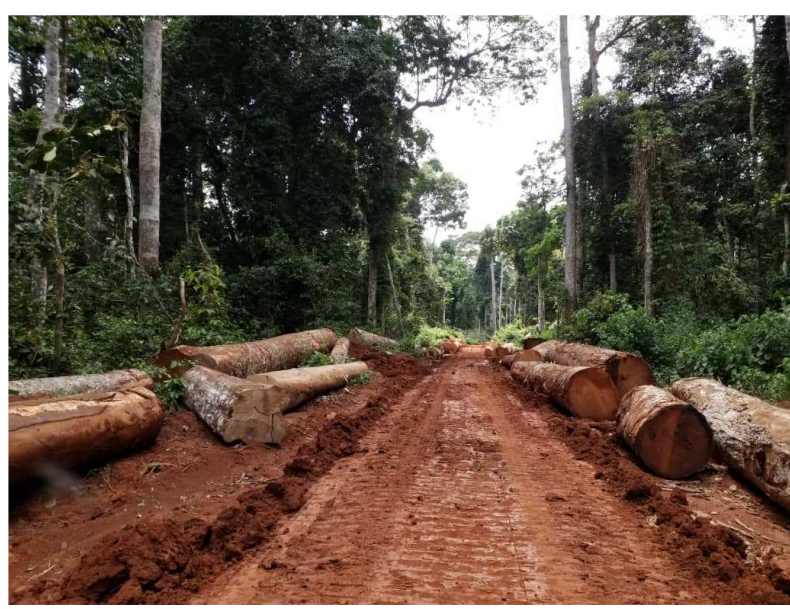

Figure 3a : Exploitation forestière au nord de la

République du Congo dans I'UFA Pokola. (Photo : Suspense Ifo, 2019).

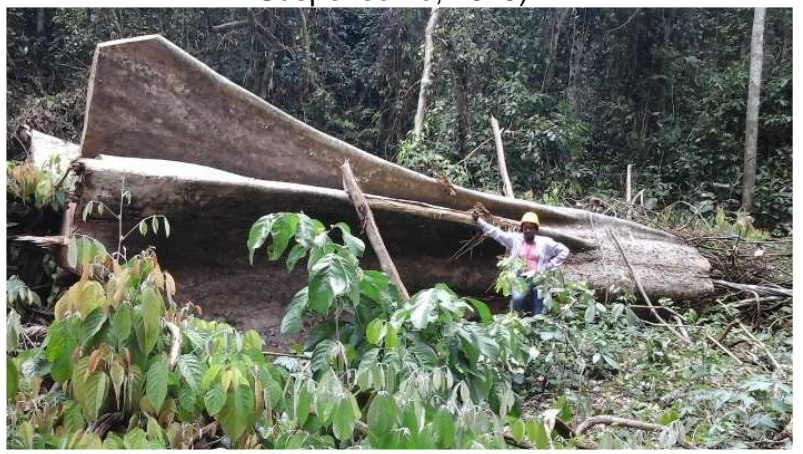

Figure 3b : Gigantisme des arbres des forêts tropicales : un arbre abattu (Ayous, ou Triplochiton scleroxylon) dans I'UFA Pokola (Photo: Suspense Ifo, 2018).

\footnotetext{
${ }^{1}$ Centre National d'Inventaire et d'Aménagement des Ressources Forestières et Fauniques (CNIAF)
} 


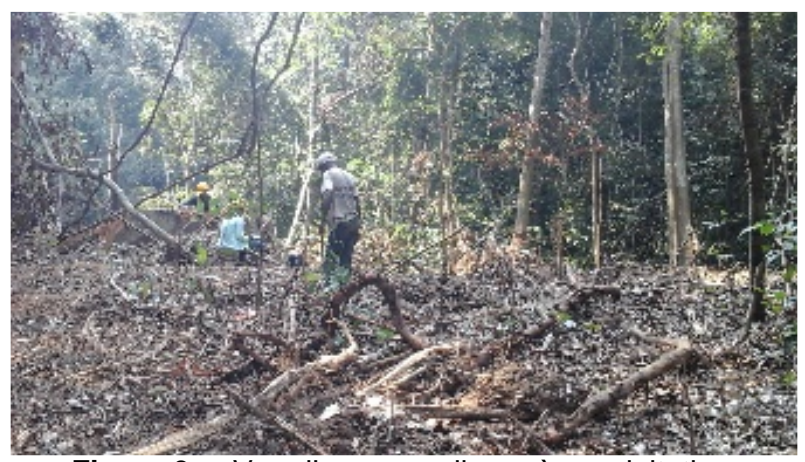

Figure 3c : Vue d'une parcelle après exploitation forestière dans la concession Mpoukou-ogooué, massif du Chaillu. (Photo : Suspense Ifo, 2018)

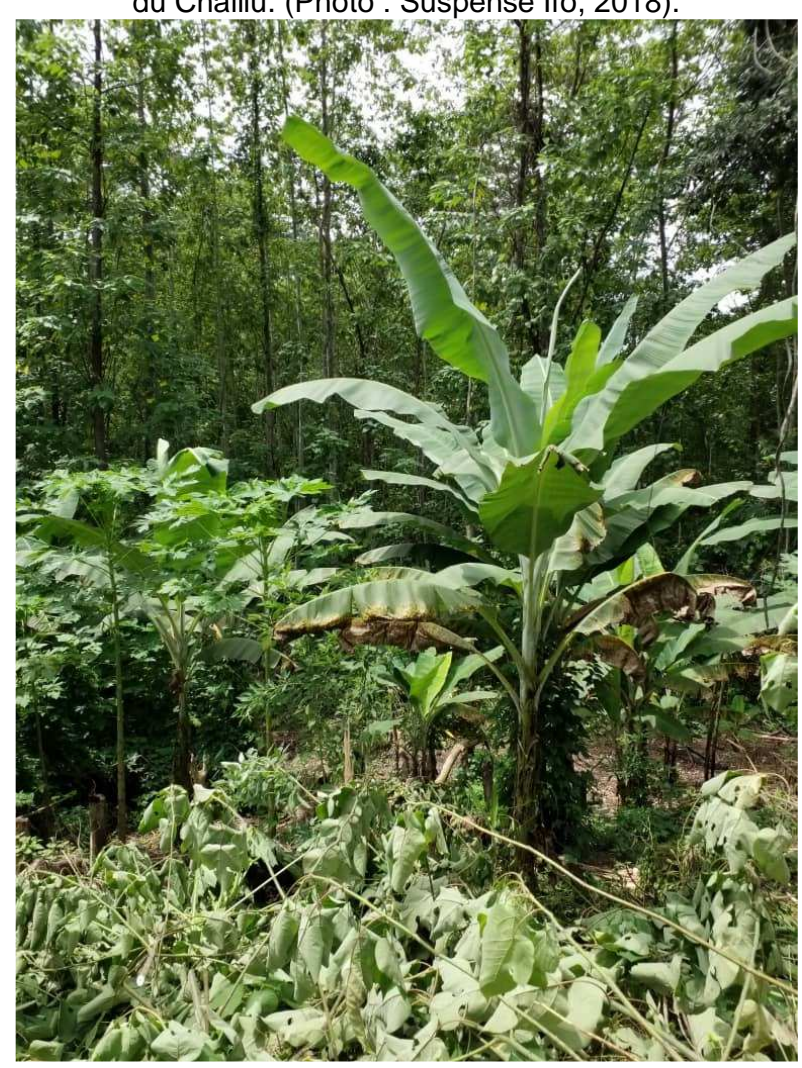

Figure 3d : Activité agricole à l'intérieur de la forêt tropicale dense du nord Congo. (Photo Suspense Ifo, 2019).

En République du Congo, la dégradation forestière semble être plus importante en comparaison à la déforestation dont les principales causes concernent l'exploitation forestière (figure 3 ), et les feux de brousse qui représentent près de $45,7 \%$ des pertes forestières.

Cette information est très importante car elle aura un impact sur le suivi du couvert forestier d'une année à une autre en raison des difficultés rencontrées par plusieurs équipes à travers le monde pour cartographier la dégradation forestière en zone tropicale. En effet, les forêts situées dans le Sud de la République du Congo sont généralement couvertes pratiquement toute l'année par des nuages très épais (FACET Congo 2012).
Avec l'engagement du pays dans le processus REDD+, la République du Congo s'est retrouvée dans le besoin de produire des cartes forestières qui présentent la situation récente des superficies forestières mais aussi la dynamique des pertes du couvert forestier entre différentes dates. L'objectif est de suivre les émissions des gaz à effet de serre (GES), mais aussi de permettre aux décideurs d'anticiper les mécanismes de réduction de ces émissions. A cet effet, la République du Congo est l'un des rares pays du bassin du Congo ayant bénéficié de plusieurs appuis cartographiques. Le pays dispose ainsi d'une multitude de produits cartographiques d'occupation du sol et forestiers couvrant la période 1990 à 2016 (FAO, UMD, CNIAF), (Ouissaka et Milandou, 2019), qui présentent des estimations différentes sur la superficie forestière, variant de 22 millions à 24 millions d'hectares, mais aussi des estimations de pertes différentes sur les mêmes périodes d'étude.

Plusieurs questions ont été soulevées par les différentes parties au niveau national concernant la qualité de ces cartes forestières produites. Ainsi, dans le but de vérifier leurs précisions et d'arriver à déterminer quelles sont les superficies cartographiques que le pays doit réellement présenter auprès de la communauté internationale, un appui méthodologique a été sollicité par le Congo auprès du projet OSFACO. Cet appui méthodologique devait assurer la qualification des cartographies nationales existantes, et proposer de nouvelles approches méthodologiques pour réduire les incertitudes dans les indicateurs de l'évolution du couvert forestier.

La mise à disposition rapide et gratuite des images satellites permet aujourd'hui de produire efficacement des cartographies forestières et de détecter les changements du couvert forestier liés à la déforestation et à la dégradation. Seulement ces cartes ne sont pas parfaites et les superficies déterminées contiennent des erreurs qui doivent être estimées (Olofsson et al., 2013).

Pour cela, une évaluation de la précision permet d'identifier les erreurs de classification des cartes et d'évaluer leurs incertitudes (GFOI, 2013 ; Olofsson et al., 2014).

L'un des moyens les plus connus d'évaluation des précisions et d'estimation des incertitudes est la comparaison des cartes classifiées avec des observations de référence (Congalton, 1991; GFOI, 2013). Cette évaluation n'est possible que si elle est basée sur une méthode d'échantillonnage (Olofsson et al., 2013 ; Sannier et al., 2014).

La République du Congo dispose d'une multitude de produits cartographiques d'occupation du sol et forestiers couvrant la période 1990 à 2016 (Ouissaka et Milandou, 2019). La méthodologie d'évaluation des précisions sera appliquée uniquement sur les cartographies forestières et les cartographies des changements du couvert forestier les plus récentes à savoir 2000-2014 et 2014-2016.

\subsection{Objectifs}

Trois objectifs sont proposés :

(i) Evaluer les superficies réelles du couvert forestier du Congo sur les années pivots 2000, 2014 et 2016, ainsi que leur niveau d'incertitude ; 
(ii) Evaluer la superficie des pertes du couvert forestier entre 2000-2014 et 2014-2016 ainsi que leur niveau d'incertitude ;

(iii) Comparer les performances des méthodes d'échantillonnage par point et surfacique pour l'estimation des superficies du couvert forestier et de son évolution.

\subsection{Zone d'étude}

La zone d'étude concerne l'ensemble du territoire congolais, situé au cœur du bassin du Congo, dont la superficie est de $342000 \mathrm{~km}^{2}$. II s'agit d'un pays où la densité de forêts représente près de $70 \%$ du territoire comme illustré en figure 4 . Selon le CNIAF, 2015, le taux de déforestation est faible et représente environ $0,05 \%$ par an. La faible pression démographique et le sousdéveloppement des infrastructures limitent la déforestation à de faibles taux annuels par rapport à des économies qui se développent plus rapidement. Cependant, le changement agro-industriel sous la forme de plantations de palmiers à huile est maintenant en expansion au Congo. Le taux de perturbation a doublé depuis 2000 pour atteindre plus de 60000 ha/an dont $43 \%$ se trouvant dans des forêts primaires (Hansen et al., 2016). Une autre caractéristique physique importante pour réaliser le suivi des forêts du sud du Congo est la présence nuageuse quasi-permanente au cours de l'an (Facet Congo 2012).

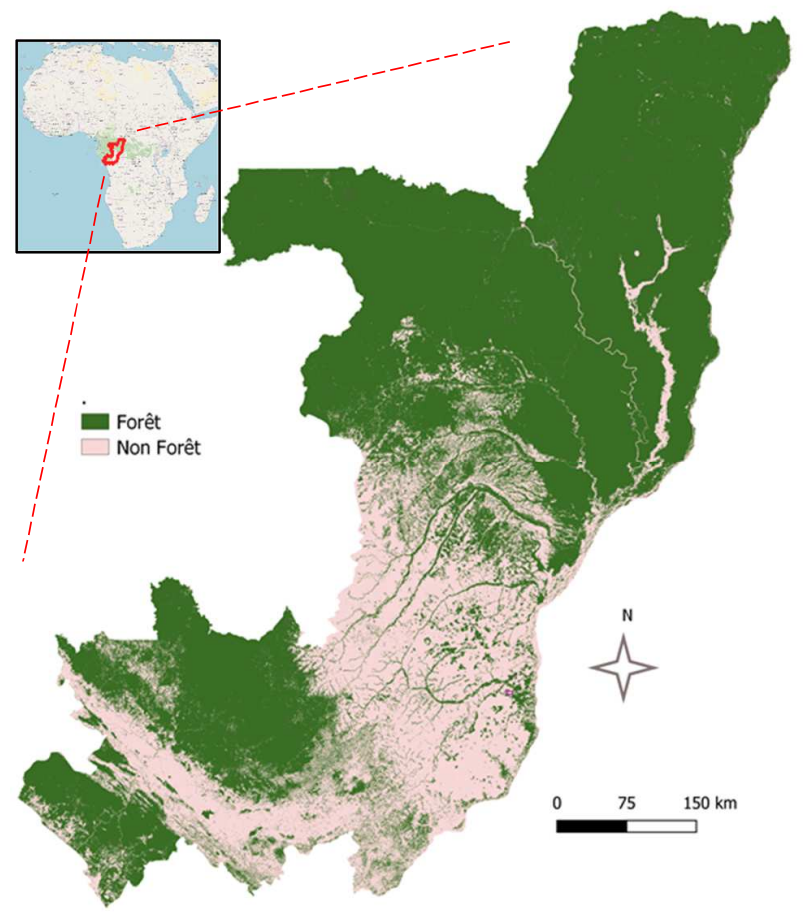

Figure 4 : Carte forestière 2016 de la République du Congo.

\section{Méthodologie}

Cette étude a impliqué une combinaison des méthodes issues des travaux de Olofsson et al., 2014 et Sannier et al., 2014 pour l'estimation des données d'activités. La méthode Olofsson est une approche par échantillonnage aréolaire où l'information est extraite au niveau du point. La méthode Sannier et al, 2014, est une approche par échantillonnage surfacique dont l'information comprend la proportion de surface pour chaque classe thématique évaluée (couvert forestier, perte du couvert forestier) et par unité d'échantillonnage de $1 \mathrm{~km}^{2}$.

Leur mise en place a permis de les comparer et de connaitre leur complémentarité. Pour ce faire les étapes suivantes ont été exécutées :

- La collecte des données cartographiques et des données sources qui ont servi à la stratification et à la mise en place du plan d'échantillonnage ;

- Les étapes de production et de photo-interprétation des échantillons nécessaires aux analyses statistiques finales.

\subsection{Collecte des données de référence et données sources}

Le projet avait à disposition plusieurs produits cartographiques couvrant la période de 1990 à 2016.

Chacun de ces produits présente des caractéristiques qui lui sont spécifiques. II s'agit de :

a) La carte de changement du couvert forestier de 1990 à 2000 et 2000 à 2010 réalisée par le CNIAF avec la société GAF ;

b) L'étendue et perte des forêts en République du Congo de 2000 à 2005 et 2005 à 2010 réalisée par le CNIAF et l'Observatoire Spatial des Forêts d'Afrique Centrale (OSFAC).

c) La carte de changement du couvert forestier en République du Congo pour la période de 2000 à 2012 réalisée par le CNIAF et la Food and Agriculture Organisation (FAO).

d) La carte du couvert forestier et des pertes de 2000 à 2014 de la République du Congo réalisée par le CNIAF et l'Université du Maryland (UMD).

e) La carte de suivi du couvert forestier en République du Congo de 2014 à 2016 réalisée par le CNIAF et I'UMD.

Devant ce nombre important de cartographies forestières, le projet a sélectionné préalablement 3 séries temporelles 2000-2012, 2000-2014 et 2014-2016.

Un travail de collecte des données couvrant l'ensemble du territoire a été mené avant le lancement de l'interprétation des échantillons. Cette collecte comprend à la fois les cartographies de références sélectionnées ainsi que l'ensemble des images satellites ayant servi à leur production.

\subsubsection{Les cartographies forestières}

Les cartographies forestières et cartographies des changements comprennent ;

i- $\quad$ Les cartes des changements, 2000-2012, 2000-2014 et 2014-2016 produites par le CNIAF et al, dont les nomenclatures respectives sont reprises dans le Tableau 1. 


\begin{tabular}{|l|l|l|}
\hline \multicolumn{1}{|c|}{$\begin{array}{c}\text { Cartographie } \\
\mathbf{2 0 0 0 - 2 0 1 2}\end{array}$} & $\begin{array}{c}\text { Cartographie } \\
\mathbf{2 0 0 0 - 2 0 1 4}\end{array}$ & $\begin{array}{l}\text { Cartographie } \\
\mathbf{2 0 1 4 - 2 0 1 6}\end{array}$ \\
\hline 0 No data & 7 Non-forêt & 7 Non-forêt \\
\hline 2- Non-forêt & 8- Eau & 8- Eau \\
\hline 4- Eau & $\begin{array}{l}\text { 1-Forêt primaire } \\
\text { sur terre ferme }\end{array}$ & $\begin{array}{l}\text { 1-Forêt primaire } \\
\text { sur terre ferme }\end{array}$ \\
\hline $\begin{array}{l}\text { 11-Forêt } \\
\text { primaire }\end{array}$ & $\begin{array}{l}\text { 2-Forêt } \\
\text { secondaire sur } \\
\text { terre ferme }\end{array}$ & $\begin{array}{l}\text { 2-Forêt } \\
\text { secondaire sur } \\
\text { terre ferme }\end{array}$ \\
\hline secondaire & $\begin{array}{l}\text { 4-Forêt primaire } \\
\text { marécageuse }\end{array}$ & $\begin{array}{l}\text { 4-Forêt primaire } \\
\text { marécageuse }\end{array}$ \\
\hline $\begin{array}{l}\text { 13-Forêt } \\
\text { primaire } \\
\text { marécageuse }\end{array}$ & $\begin{array}{l}\text { 5-Forêt } \\
\text { secondaire } \\
\text { marécageuse }\end{array}$ & $\begin{array}{l}\text { 5-Forêt } \\
\text { secondaire } \\
\text { marécageuse }\end{array}$ \\
\hline & $\begin{array}{l}\text { 3-Plantations } \\
\text { forestières }\end{array}$ & $\begin{array}{l}\text { 3-Plantations } \\
\text { forestières }\end{array}$ \\
\hline $\begin{array}{l}\text { 6- Autres zones } \\
\text { marécageuses }\end{array}$ & \\
\hline $\begin{array}{l}\text { 31- Perte en } \\
\text { forêt primaire }\end{array}$ & $\begin{array}{l}\text { 9- Perte en forêt } \\
\text { primaire sur } \\
\text { terre ferme }\end{array}$ & $\begin{array}{l}\text { 9- Perte en forêt } \\
\text { primaire sur } \\
\text { terre ferme }\end{array}$ \\
\hline $\begin{array}{l}\text { 34- Perte en } \\
\text { forêt primaire } \\
\text { sur route }\end{array}$ & $\begin{array}{l}\text { 13- Perte en } \\
\text { plantation } \\
\text { forestière }\end{array}$ & $\begin{array}{l}\text { 11- Perte en } \\
\text { plantation } \\
\text { forestière }\end{array}$ \\
\hline $\begin{array}{l}\text { 32- Perte en } \\
\text { forêt secondaire }\end{array}$ & $\begin{array}{l}\text { 10- Perte en } \\
\text { forêt secondaire } \\
\text { sur terre ferme }\end{array}$ & $\begin{array}{l}\text { 10- Perte en } \\
\text { forêt secondaire } \\
\text { sur terre ferme }\end{array}$ \\
\hline $\begin{array}{l}\text { 33- Perte en } \\
\text { forêt primaire } \\
\text { marécageuse } \\
\text { forêt primaire } \\
\text { marécageuse } \\
\text { forêt primaire }\end{array}$ \\
\hline
\end{tabular}

\begin{tabular}{|l|l|l|}
\hline $\begin{array}{c}\text { Cartographie } \\
\mathbf{2 0 0 0 - 2 0 1 2}\end{array}$ & $\begin{array}{c}\text { Cartographie } \\
\mathbf{2 0 0 0 - 2 0 1 4}\end{array}$ & $\begin{array}{c}\text { Cartographie } \\
\mathbf{2 0 1 4 - 2 0 1 6}\end{array}$ \\
\hline & $\begin{array}{l}\text { 12- Perte en } \\
\text { forêt secondaire } \\
\text { marécageuse }\end{array}$ & $\begin{array}{l}\text { 13- Perte en } \\
\text { forêt secondaire } \\
\text { marécageuse }\end{array}$ \\
\hline & $\begin{array}{l}\text { 14 Perte autres } \\
\text { marécages }\end{array}$ & \\
\hline
\end{tabular}

Tableau 1 : nomenclatures des cartographies forestières sélectionnées.

La méthodologie utilisée est celle du «Wall to Wall » développée par l'Université du Maryland. Cette méthodologie automatisée utilise la Classification Hiérarchique de «l'Arbre de décision » (Decision Trees), CNIAF, 2015. Toutefois, de manière générale, les cartes produites comportent des erreurs et comme spécifié maintenant dans de nombreux articles et rappelé dans GFOI (2013), il n'est pas recommandé d'extraire directement les statistiques de superficie à partir de ces cartes.

ii- $\quad$ Les données auxiliaires utilisées en complément. II s'agit par exemple des limites de concessions forestières, des voies de communication, du réseau hydrologique et des zones de plantations forestières.

\subsubsection{Les images satellites sources}

Plus de 150 images satellites, illustrées dans la figure 5, ont été collectées afin d'obtenir les 3 années de référence (pivots) complètes sur l'ensemble du territoire.

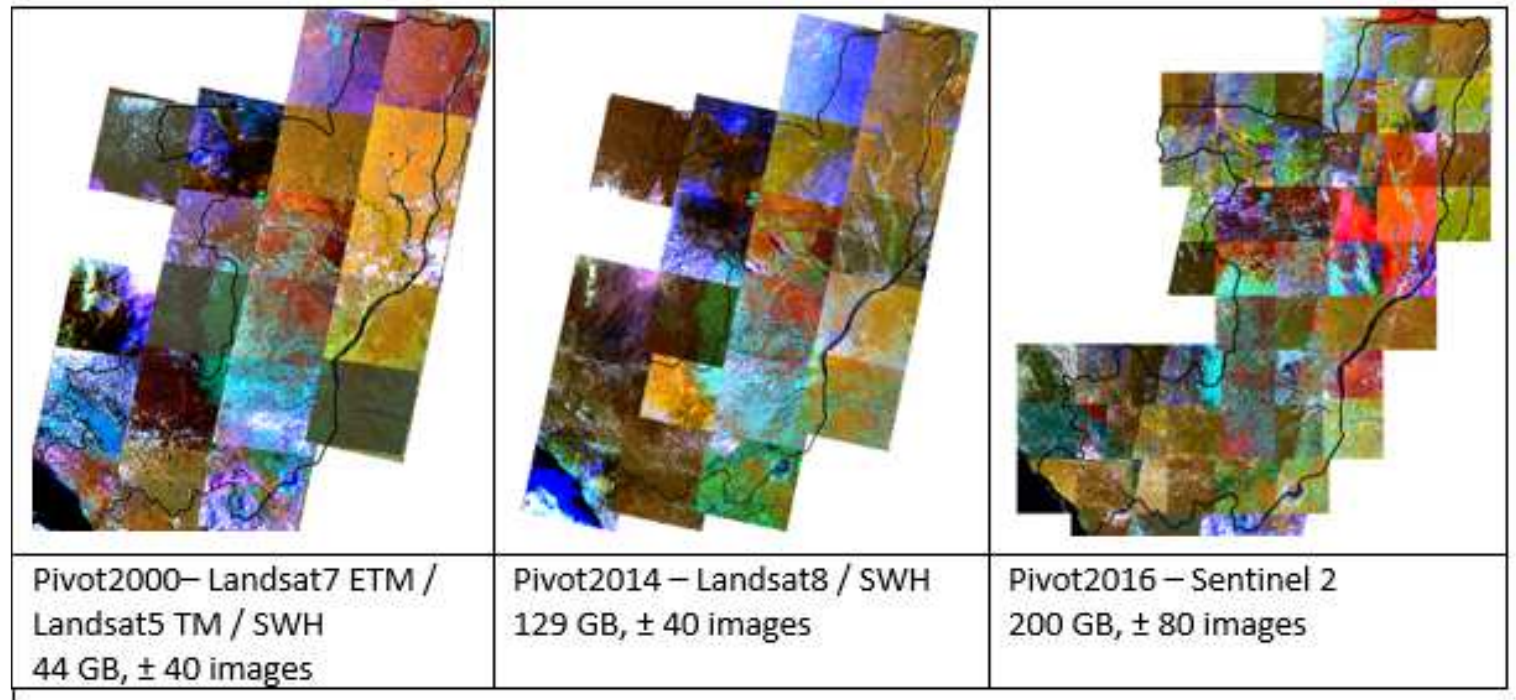

Figure 5 : aperçu des couvertures en images satellites des 3 pivots.

La couverture nationale du pivot 2000 est constituée d'une quarantaine d'images Landsat 7 ETM, de $30 \mathrm{~m}$ de résolution spatiale, acquises entre 1999 et 2002 et d'un complément en images SPOT4 à $20 \mathrm{~m}$ de résolution spatiale, acquises entre 1998 et 2008, issues du service
Spot World Heritage (SWH) qui met à disposition gratuitement les images d'archive ortho-rectifiées des satellites SPOT1 à SPOT5.

La couverture nationale du pivot 2014 est constituée d'une quarantaine d'images landsat 8 , de $30 \mathrm{~m}$ de résolution 
spatiale, acquises entre 2014 et 2015 et d'un complément d'images SPOT4/SPOT5 $(20 \mathrm{~m}$ et $10 \mathrm{~m}$ de résolution spatiale) issues du service SPOT World Heritage (SWH). Enfin, la couverture nationale du pivot 2016 est constituée de plus d'une soixante-dizaine d'images Sentinel 2, de $10 \mathrm{~m}$ de résolution spatiale, acquise entre 2015 et 2017 .

En ce qui concerne l'année 2012, le projet ne disposait pas de la couverture d'images satellites source de $2012 \pm 1$ an. Pour pallier ce manque, l'interprétation des points a été établie sur la base des images Google Earth en utilisant la fonction recherche d'image d'archive Google Earth.

La forte nébulosité présente sur la région sud de la République du Congo une grande partie de l'année a contraint de sélectionner une quantité importante d'images satellites sur cette région.

\subsection{Travaux préparatoires et pré-évaluation de la précision des cartes 2000- 2012 et 2000-2014}

\subsubsection{Taille et choix de l'échantillonnage}

Sur la base d'un échantillonnage stratifié aléatoire par point, une première sélection de 1000 échantillons sur tout le territoire, (figure 7) a été générée et interprétée.

La méthode d'échantillonnage aléatoire stratifié a été choisie car elle est robuste et permet de réduire au maximum le biais associé à l'échantillonnage. En effet, d'après Olofsson et al., 2014, l'échantillonnage aléatoire stratifié est un plan pratique qui répond aux objectifs d'évaluation de la précision de base de données géographiques et thématiques.

Cet échantillonnage offre la possibilité d'augmenter la taille de l'échantillon dans les strates qui occupent une faible proportion de la superficie et c'est l'un des modèles les plus faciles à mettre en œuvre.

La pré-évaluation des précisions a été réalisée initialement sur les cartographies forestières 2000-2012 et 2000-2014, la dernière 2014-2016 n'étant pas encore disponible au moment de ces travaux préparatoires.

\subsubsection{Réalisation des matrices de confusion}

L'interprétation des 1000 points décrits précédemment a permis de réaliser une première évaluation de la précision des cartographies forestières afin de déterminer celles qui devaient être retenues pour l'estimation des données d'activités du Congo. Pour ce faire, deux matrices de confusion, à base des cartes de changements 2000-2012, et 2000-2014 ont été réalisées et sont représentées respectivement dans les tableaux 2 et 3 .

\begin{tabular}{|c|c|c|c|c|c|c|c|}
\hline & \multirow[b]{2}{*}{ 2000-2012 } & \multicolumn{4}{|c|}{ REFERENCE } & \multirow[b]{2}{*}{$\begin{array}{l}\text { Précision } \\
\text { utilisateur }\end{array}$} & \multirow[b]{2}{*}{$\begin{array}{c}\text { Commis } \\
\text { sion }\end{array}$} \\
\hline & & 1-Forêt & $\begin{array}{r}\text { 2-Non } \\
\text { forêt }\end{array}$ & 3-Perte & $\begin{array}{l}\text { Total } \\
\text { général }\end{array}$ & & \\
\hline \multirow{6}{*}{ 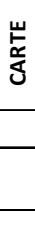 } & 1-Forêt & 692,06 & 21,51 & 1,20 & 714,77 & $\mathbf{9 7 \%}$ & $3 \%$ \\
\hline & 2-Non Forêt & 5,38 & 275,42 & & 280,80 & $98 \%$ & $2 \%$ \\
\hline & 3-Perte & 1,75 & 0,44 & 1,88 & 4,07 & $46 \%$ & $54 \%$ \\
\hline & Total général & 699,19 & 297,37 & 3,08 & 999,64 & & \\
\hline & \begin{tabular}{|l|} 
Précision \\
producteur
\end{tabular} & $99 \%$ & $93 \%$ & $61 \%$ & & & \\
\hline & Omission & $1 \%$ & $7 \%$ & $39 \%$ & & & \\
\hline
\end{tabular}

Tableau 2 : Matrice de confusion pondérée entre la carte 2000-2012 et les points de validation.

\begin{tabular}{|c|c|c|c|c|c|c|c|}
\hline & \multirow[b]{2}{*}{$2000-2014$} & \multicolumn{4}{|c|}{ REFERENCE } & \multirow[b]{2}{*}{$\begin{array}{l}\text { Précision } \\
\text { utilisateur }\end{array}$} & \multirow[b]{2}{*}{$\begin{array}{c}\text { Commis } \\
\text { sion }\end{array}$} \\
\hline & & 1-Forêt & $\begin{array}{r}\text { 2-Non } \\
\text { forêt }\end{array}$ & 3-Perte & $\begin{array}{c}\text { Total } \\
\text { général }\end{array}$ & & \\
\hline \multirow{3}{*}{ 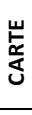 } & 1-Forêt & 688,46 & 22,59 & 1,14 & 712,18 & $97 \%$ & $3 \%$ \\
\hline & 2-Non Forêt & 6,52 & 274,47 & 0,13 & 281,11 & $98 \%$ & $2 \%$ \\
\hline & 3-Perte & 4,22 & 0,31 & 1,82 & 6,35 & $29 \%$ & $71 \%$ \\
\hline & Total général & 699,19 & 297,37 & 3,08 & 999,64 & & \\
\hline & $\begin{array}{l}\text { Précision } \\
\text { producteur }\end{array}$ & 98\% & $92 \%$ & $59 \%$ & & & \\
\hline & Omission & $2 \%$ & $8 \%$ & $41 \%$ & & & \\
\hline
\end{tabular}

Tableau 3 : Matrice de confusion pondérée entre la carte 2000-2014 et les points de validation.

Les tableaux 2 et 3 représentent les matrices de confusion pondérées en prenant en compte les différences d'intensité d'échantillonnage pour chacune des strates issues de l'analyse des 1000 échantillons interprétés. C'est pourquoi les chiffres présentés ne sont pas entiers mais représentent la somme des échantillons pondérés dans chaque combinaison représentée dans les matrices. Les colonnes indiquent les résultats des données de référence (points) et en ligne les résultats de la cartographie. Les matrices révèlent en bas de la colonne (encadré rouge), qu'il y a $39 \%$ d'omission dans la classe perte pour la carte et les changements $2000-2012$ et $41 \%$ d'omission dans la classe perte pour la carte et les changements 2000-2014. Ces deux cartes sont donc assez comparables avec un niveau d'omission des pertes du couvert forestier assez important de l'ordre de $40 \%$, qui justifie une approche complémentaire pour définir une strate d'omission spécifique afin de réduire les incertitudes de l'estimation de la superficie des pertes de couvert forestier. Les cartes présentent aussi des erreurs substantielles en termes de contamination (ou commission), de l'ordre de $54 \%$ et jusqu'à $71 \%$ respectivement pour les cartes 2000-2012 et 2000-2014. Toutefois, les erreurs de contamination sur la strate de changement ont un impact moindre que les erreurs d'omission sur l'efficacité de la stratification adoptée et sur le calcul des incertitudes qui en résultent. En effet des erreurs d'omission sur la définition de la strate de changement accroissent de manière substantielle l'intervalle de confiance des estimations de superficies de pertes de couvert forestier.

Dans le but de maintenir la chronologie des données, et compte tenu de la proximité des valeurs d'omissions entre les pertes 2000-2012 et 2000-2014, l'analyse et la consolidation finale des résultats sur les estimations de superficie et des pertes du couvert forestier a été poursuivie sur les cartographies forestières les plus récentes, c'est-à-dire 2000-2014 et 2014-2016.

\subsection{Stratification et génération du plan d'échantillonnage}

Pour améliorer la caractérisation des zones d'omission, un jeu de données externes a été analysé et utilisé pour la stratification. Cela concerne les réseaux de communication (hydrographie et routes, pistes), les bordures des zones de perte du couvert forestier déjà existantes, les bordures des concessions forestières, etc. Les deux cartes 2000-2014 et 2014-2016 ont été reclassées en cartographie trinaire forêt, non-forêt et 
pertes, puis ces deux cartes ont été fusionnées pour obtenir une nouvelle carte contenant 4 strates à savoir :

La strate Forêt stable entre 2000-2014-2016 ;

La strate Non-forêt stable entre 2000-2014-2016 ;

La strate perte du couvert entre 2000-2014 ;

- $\quad$ La strate perte du couvert entre 2014-2016.

Les principales étapes de la stratification ayant donné lieu au plan d'échantillonnage sont reprises dans la figure 6 :
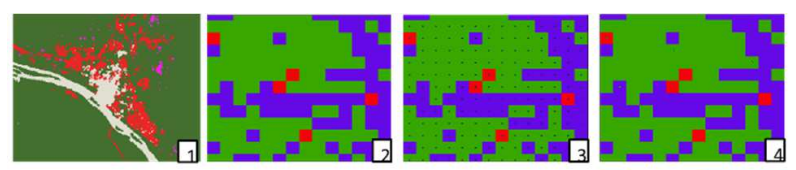

1: Stratification (résolution $30 \mathrm{~m}$ )

2: Ré-échantillonnage de la stratification à $1000 \mathrm{~m}$ résolution 3 : Conversion du raster en points (chaque points à la valeur du pixel correspondant)

4 : Tirage proportionnel de 1000 échantillons en fonction de la superficie de chaque strate

5 : Conversion des points en segment de $1000 \times 1000 \mathrm{~m}$

Figure 6 : Principales étapes de la stratification.

Les cartographies forestières sont fournies au format GEOTIF avec un pixel de $30 \mathrm{~m} \times 30 \mathrm{~m}$ correspondant à la taille du pixel Landsat.

L'objectif du rééchantillonnage est d'obtenir des cellules d'1 km² correspondant à la taille de l'échantillon de référence. Cette surface d' $1 \mathrm{~km}^{2}$ est un compromis entre un temps de production manuel et une taille suffisamment représentative du paysage du territoire, afin d'éviter de n'avoir que des valeurs à 0 ou $100 \%$ de couvert arboré si la surface est trop petite ou un temps de production trop important si celle-ci est trop grande, sur la base des critères définis par Gallego (1995). Une grille de $1 \mathrm{~km}$ a donc été superposée aux cartes thématiques à $30 \mathrm{~m}$ disponibles sur les deux périodes d'observation. Dans un premier temps toutes les cellules de la grille qui contenaient des pertes de couvert dans une des deux cartes ont été attribuées à la strate de perte de couvert forestier correspondante (2000-2014 ou 2014-2016). Dans un second temps toutes les cellules couvertes sur au moins $30 \%$ de leur superficie par de la forêt ont été attribuées à la strate forêt stable. Les cellules restantes ont été attribuées à la strate non-forêt stable.

Le nombre d'échantillons tirés par strate est proportionnel à sa superficie. La sélection des 1000 échantillons est faite de façon aléatoire orientée ce qui permet d'obtenir un plan d'échantillonnage spatialement représentatif à la fois des différentes strates mais aussi ayant une bonne distribution sur l'ensemble du pays. La figure 7 illustre le plan d'échantillonnage final. Le tableau 5 propose le nombre exact d'échantillon dans les 4 strates, déterminé selon le poids de chacune d'entre elles:

\begin{tabular}{|c|c|c|c|}
\hline $\begin{array}{c}\text { Code } \\
\text { strate }\end{array}$ & $\begin{array}{c}\text { Nom de } \\
\text { strate }\end{array}$ & $\begin{array}{c}\text { Superficie } \\
\text { (ha) }\end{array}$ & $\begin{array}{c}\text { Nombre } \\
\text { d'échantillons }\end{array}$ \\
\hline 1 & Forêt & 22592300 & 640 \\
\hline 2 & Non Forêt & 7715900 & 225 \\
\hline 3 & $\begin{array}{c}\text { Perte 2000 } \\
-2014\end{array}$ & 2918300 & 85 \\
\hline
\end{tabular}

\begin{tabular}{|c|c|r|c|}
\hline 4 & $\begin{array}{c}\text { Perte 2014 } \\
-2016\end{array}$ & 1021000 & 50 \\
\hline Total & & $\mathbf{3 4 2 4 7 5 0 0}$ & $\mathbf{1 0 0 0}$ \\
\hline
\end{tabular}

Tableau 4 : Répartition des échantillons par strate.

La première analyse du tableau 4 montre que la strate «Forêt» par exemple étant la plus représentative, concentre un maximum d'échantillons contrairement à la strate «Perte du couvert 2014-2016» qui par sa faible superficie contient peu d'échantillon. Le minimum d'échantillon requis pour chacune des strates a toutefois été fixé à 50 afin de garantir une représentativité spatiale suffisante.

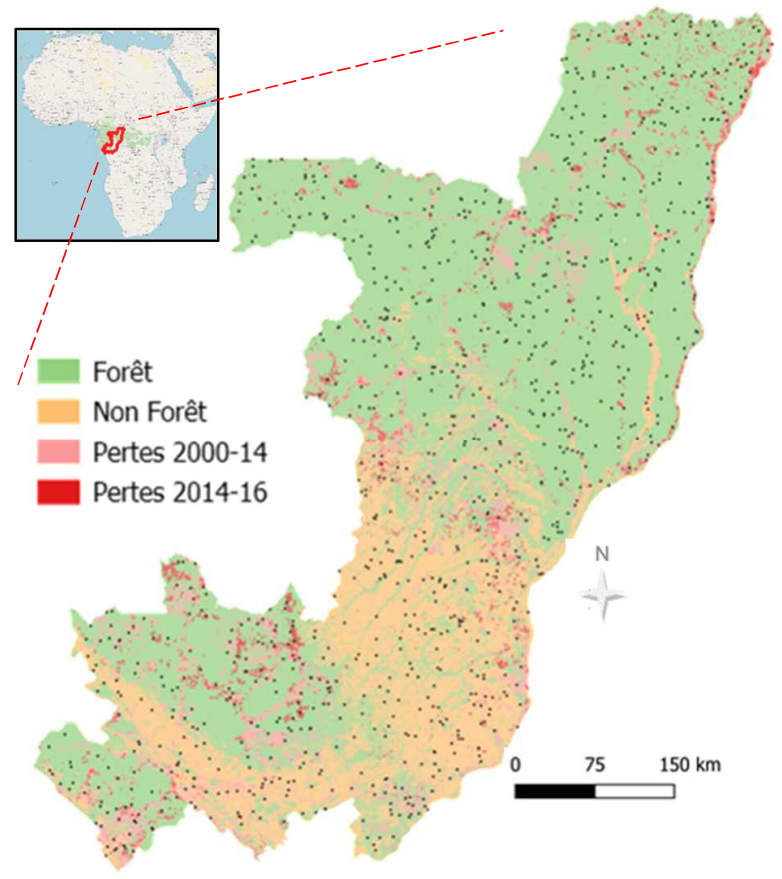

Figure 7 : Aperçu de la distribution finale des échantillons par strates sur le territoire congolais.

\subsection{Production des échantillons}

\subsubsection{Photo-interprétation des échantillons}

La méthode de production des échantillons des différents pivots et des changements survenus entre 2000-2014 et 2014-2016 est basée sur une technique de photointerprétation visuelle et de délimitation manuelle des objets. La méthodologie de production simplifiée est illustrée dans la figure 8. Les 1000 échantillons ou segments de $1 \mathrm{~km}^{2}$ ont été distribués puis photointerprétés par une équipe indépendante de 4 experts cartographes. L'interprétation est réalisée sur le logiciel libre QGIS. Une table attributaire est associée à la base de données vectorielle contenant l'information sur les classes d'occupation du sol et les surfaces. Les échantillons par point ont été générés à partir des échantillons surfaciques en prenant la classe thématique correspondant au centre de l'échantillon surfacique. 


\section{Echantillon PSU N699 (X=1 738300 Y=-23 700, EPSG:3857).}

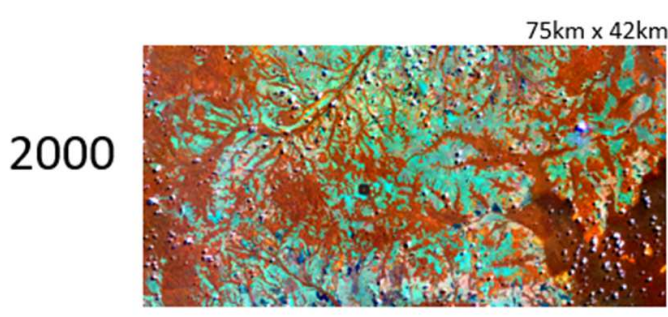

Landsat 7 ETM, 2000 ( Rés. $=30 \mathrm{~m}$ )

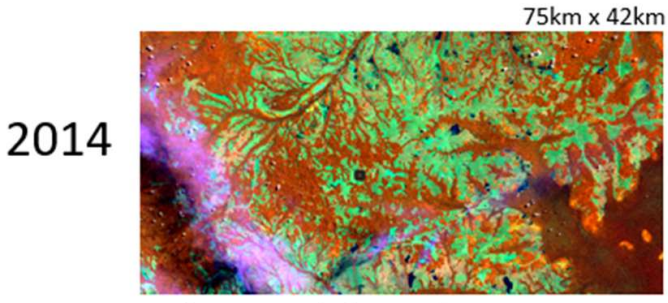

Landsat 8, 2014 ( Rés. $=30 \mathrm{~m}$ )

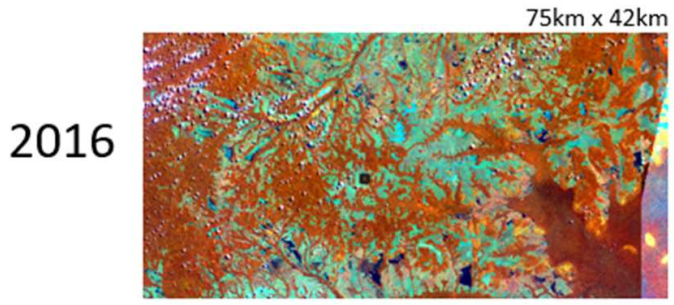

Sentinel2, 2016 ( Rés. $=10 \mathrm{~m}$ )

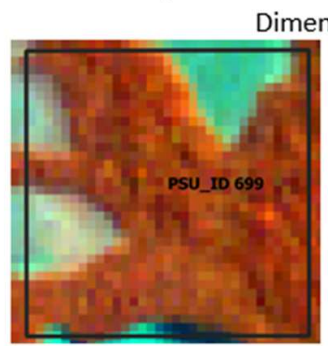

Landsat7, 2000 (Rés. =30m)

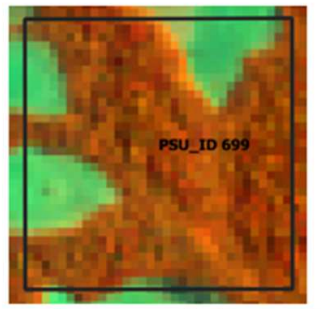

Landsat 8, 2014 ( Rés. $=30 \mathrm{~m}$ )

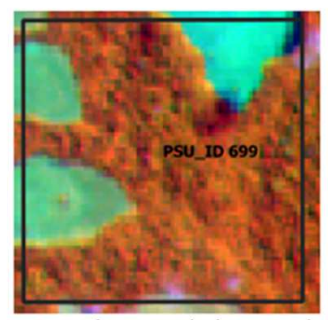

Sentinel2, 2016 (Rés. =10m)

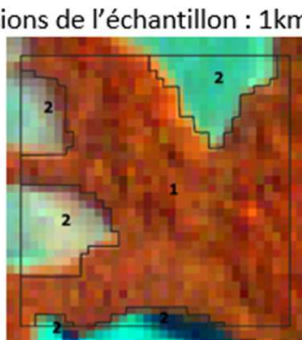

Contour vecteur en 2000.

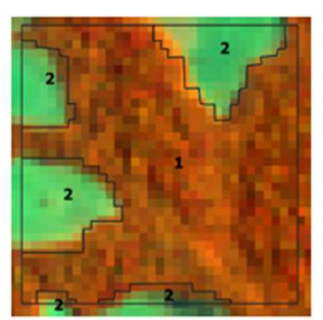

Contour vecteur en 2014

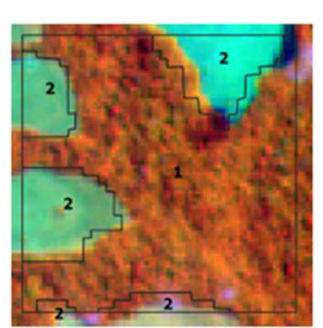

Contour vecteur en 2016

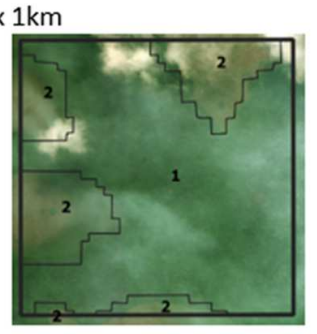

Données auxiliaires Google Earth, Bing Aerial

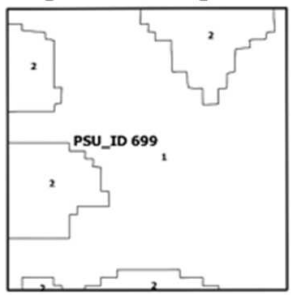

Information vecteur et table attributaire associée Classe 1 : Forêt, Classe2 : Non foret

Figure 8 : Méthode de cartographie par photo-interprétation des échantillons.

La nomenclature et les définitions des classes sont représentées dans le tableau 5 et comprennent 4 classes principales d'occupation du sol: Forêt, non-forêt, eau, plantations forestières et nuages. La définition de la forêt utilisée est celle qui a été officiellement adoptée par le Congo en mars 2014 (rapport d'atelier sur la définition forêt, CN-REDD 2014b), appliquée à l'échelle nationale et contenue dans le NERF² 2016. Elle précise que « la forêt est un espace couvrant une superficie minimale de 0,5 hectare $(0,50 \mathrm{ha})$, avec des arbres ayant une hauteur minimale de 3 mètres $(3 \mathrm{~m})$ et un taux minimal de couverture de houppier de $30 \% »$.

2 NERF : Niveau des Emissions de Référence pour les Forêts de la République du Congo

\begin{tabular}{|c|c|c|}
\hline Classe & code & Définition \\
\hline Forêt & 1 & $\begin{array}{l}\mathrm{MMU} \geq 0,5 \text { ha Densité } \geq 30 \% \mathrm{H} \geq 5 \mathrm{~m} \\
=\text { Terrain forestier (forêts denses, forêts } \\
\text { secondaires, forêts marécageuses et les } \\
\text { mangroves). }\end{array}$ \\
\hline Non forêt & 2 & $\begin{array}{l}\text { Autre terrain non forestier (urbain, routes, les } \\
\text { savanes arbustives herbeuses (densité d'arbre } \\
<25-30 \% \text {, sols nus, agricultures) }\end{array}$ \\
\hline Eau & 3 & $\mathrm{MMU} \geq 0,5 \mathrm{ha}$ \\
\hline $\begin{array}{r}\text { Plantations } \\
\text { forestières }\end{array}$ & 4 & $\begin{array}{l}\text { Plantations forestières comprennent les } \\
\text { essences plantées d'arbres telles que les } \\
\text { Eucalyptus, Pinus Sp, sapins, les palmiers ...) }\end{array}$ \\
\hline Nuages & 9 & $\begin{array}{l}\text { Si présence de nuages sur toutes les images du } \\
\text { pivot couvrant l'echantillon. }\end{array}$ \\
\hline
\end{tabular}

Tableau 5 : Nomenclature et définition des classes.

\subsubsection{Détection des changements}

La base de changement est produite par interprétation visuelle et délimitation manuelle des changements en comparant l'image de 2000 avec celle de 2014 puis en comparant l'image de 2014 avec celle de 2016. Seuls les changements liés à la perte du couvert forestier d'une 
superficie supérieure ou égale à 0,5 ha ont été pris en compte et délimités manuellement.

Un point important à noter est qu'un changement d'occupation du sol (OCS) n'entraine pas forcément un changement d'affectation ou d'utilisation des terres. Selon Lambin et al, 2003, deux types de changement d'occupation des terres sont définis : les conversions et les modifications. La conversion de la couverture terrestre est considérée comme un remplacement complet d'un type de couverture par un autre et est mesurée par le passage d'une catégorie de couverture terrestre à une autre comme c'est le cas d'une expansion agricole, d'une extension urbaine, d'une déforestation. Les modifications de la couverture terrestre sont des changements plus subtils qui affectent le caractère de la couverture terrestre sans modifier sa classification globale. C'est le cas d'une dégradation progressive de la couverture forestière liée à une coupe sélective d'arbres et qui reste en usage forêt.
Les changements pris en compte dans notre projet intègrent les conversions et «certaines modifications importantes " du couvert forestier, à partir du moment où le changement du couvert forestier est considéré comme observable et réel sur l'image satellite. Si un doute subsiste dans le changement observé, il n'est pas cartographié et la classe d'OCS restera identique aux deux dates. II est donc évident que les changements identifiés sont totalement dépendants des données sources d'observation c'est-à-dire des images satellites et de leurs résolutions spatiales, spectrales et temporelles. Par conséquent certains changements subtils notamment les modifications au sens Lambin et al, 2003 risquent d'être sous-estimés.

Un exemple de changement du couvert forestier pris en compte est illustré ci-dessous dans la figure 9 . 


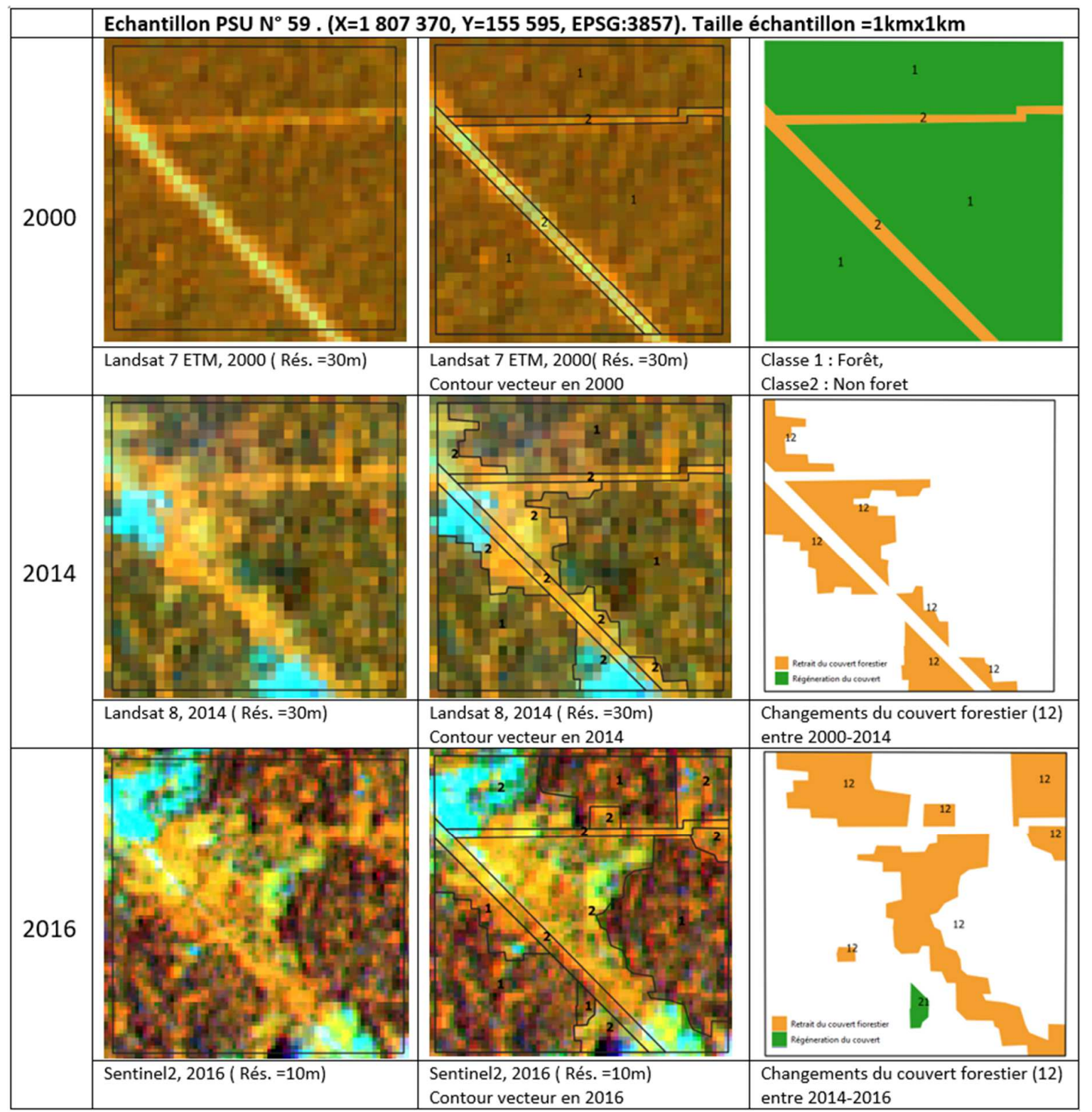

Figure 9 : Cartographie des changements du couvert forestier entre 2000, 1014 et 2016 sur un échantillon (Près de la ville de Mboko).

\subsection{Production des estimateurs}

Un estimateur est une statistique permettant d'évaluer un paramètre inconnu d'une population relatif à une loi de probabilité. Dans le cadre de cette étude, les variables à estimer sont la proportion du territoire couvert par les forêts au Congo ainsi que par les données d'activités y afférentes pour la période 2000-2014-2016.

Pour ce faire la méthodologie développée a permis de tester l'estimateur direct qui n'utilise pas l'information de la carte (mais uniquement les échantillons) et l'estimateur de Régression Assisté par Modèle (MAR) qui, en plus des échantillons, utilise la carte pour réduire l'incertitude et améliorer la précision des estimateurs dans la mesure où la carte utilisée est de bonne qualité avec une précision thématique élevée.

\subsubsection{Estimateurs directs}

Les estimateurs directs ont été utilisés avec la référence au niveau du segment pour estimer les moyennes de population pour les deux variables de réponse :

(1) Proportion de forêt pour laquelle $\mathrm{z}_{\mathrm{i}}=\mathrm{y}_{\mathrm{i}}^{\text {ref,t }}$ est l'observation de référence pour $i \varepsilon S_{i}$ pour la tième année de référence,

(2) Proportion nette de déforestation pour laquelle $\mathrm{Z}_{\mathrm{i}}=$ $y_{i}{ }^{\text {ref,t2 }}-y_{i}^{\text {ref,t1 }} i$ est l'observation de référence pour i $\varepsilon S_{i}$ pour l'intervalle $t_{1}$ à $t_{2}$. Ainsi, l'équation appliquée pour l'estimateur direct prend la forme de : 


$$
\begin{array}{lr}
\text { Moyenne (1) } & \text { Variance (2) } \\
\hat{\mu}=\frac{1}{\mathrm{~m}} \sum_{\mathrm{i} \varepsilon \mathrm{S}_{\mathrm{I}}} \mathrm{z}_{\mathrm{i}} & \operatorname{Vâr}(\hat{\mu})=\frac{1}{\mathrm{~m}(\mathrm{~m}-1)}
\end{array}
$$

Avec : $m=$ le nombre de segments, $\mathrm{z}_{\mathrm{i}}=\operatorname{yref}_{i} \mathrm{t}^{\mathrm{t}}-\mathrm{yref}_{i} \mathrm{t}^{1}$ superficie d'une entité à une date donnée

Ces estimateurs sont appliqués pour chaque strate et les superficies de chaque strate sont additionnées afin d'obtenir l'estimation globale à l'échelle du Congo. Le calcul de la variance suit le même principe et correspond à la somme du produit du carré des superficies avec la variance des proportions pour chacune des strates.

\subsubsection{Estimateurs de Régression Assistée (MAR)}

Les estimateurs MAR (Model Assisted Regression) ont été utilisés avec la combinaison des références au niveau du segment et données cartographiques pour estimer les moyennes de population pour les deux variables de réponse :

(1) proportion forêt pour laquelle $z_{i}=y_{i}{ }^{\text {ref,t2 }}$ est l'observation de référence pour i $\varepsilon S_{i}$ pour la tième année et $z_{i}{ }^{\text {map,t }}$ est la prédiction cartographique correspondante, et (2) proportion nette de déforestation pour laquelle $\mathrm{z}_{\mathrm{i}}=\mathrm{y}_{\mathrm{i}}{ }^{\text {ref,t2- }}$ $y_{i}{ }^{\text {ref,t1 }}$ est l'observation de référence pour i $\varepsilon S_{i}$ pour l'intervalle $t_{1}$ à $t_{2}$ et $z_{i}=y_{i}$ mapt2-ymap,t1 est la prédiction cartographique correspondante. L'estimateur initial de la moyenne de la population est :

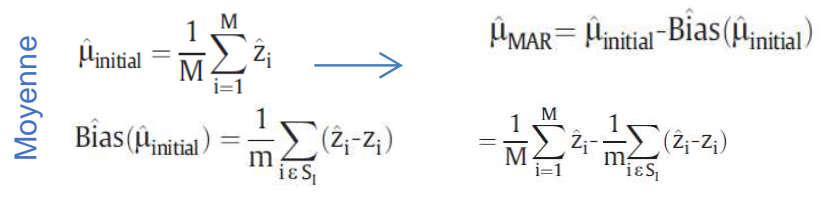

Un estimateur de la variance de $\hat{u}^{\mathrm{MAR}}$ est:

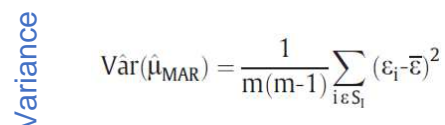

Avec $\varepsilon_{\mathrm{i}} \hat{z}_{\mathrm{i}}-\mathrm{z}_{\mathrm{i}}$ and $\bar{\varepsilon}=\frac{1}{\mathrm{~m}} \sum_{\mathrm{i} \varepsilon \mathrm{S}_{\mathrm{i}}} \varepsilon_{\mathrm{i}}$

De cette façon, la proportion de la couverture forestière est estimée pour chaque année de référence, et la proportion nette de déforestation est estimée pour chaque intervalle de temps.

Ces estimateurs sont appliqués pour chaque strate et les estimateurs à l'échelle du pays sont calculés selon les mêmes principes que pour l'estimateur direct.

\section{Résultats}

Les tableaux 6 et 7 fournissent l'ensemble des surfaces en hectares, des précisions et des intervalles de confiance pour chaque état du couvert forestier et de ses pertes survenues entre 2000-2014 et 2014-2016, pour l'approche ponctuelle et surfacique à partir des échantillons définis dans les sections 2.2 et 2.3 .

\subsection{Estimations 2000-2014}

\begin{tabular}{|c|c|c|c|c|c|c|}
\hline & \multicolumn{2}{|c|}{ Échantillons surfaciques 2000-2014 } & \multicolumn{3}{|c|}{ Échantillons ponctuels 2000-2014 } \\
(ha)
\end{tabular}

Tableau 6 : Estimations 2000-2014 selon les échantillons surfaciques et ponctuels.

Selon l'estimateur direct, la superficie du couvert forestier du Congo en 2014 est de l'ordre de 23,10 $\pm 0,46$ millions d'ha contre 23,23 $\pm 0,20$ millions d'ha selon l'estimateur de régression. L'estimateur le plus précis est celui obtenu via le modèle de régression car son intervalle de confiance est nettement plus faible.

Concernant les pertes du couvert forestier entre 2000 et 2014, l'estimateur direct présente une perte de 318175 ha \pm 93000 ha alors que l'estimateur de régression estime la

\begin{tabular}{|c|c|c|c|c|c|c|}
\hline & \multicolumn{3}{|c|}{\begin{tabular}{|c|} 
Échantillons surfaciques 2014-2016 \\
(ha)
\end{tabular}} & \multicolumn{3}{|c|}{$\begin{array}{l}\text { Échantillons ponctuels 2014-2016 } \\
\text { (ha) }\end{array}$} \\
\hline & \begin{tabular}{|l|} 
Couvert \\
Forêt 2014
\end{tabular} & $\begin{array}{l}\text { Couvert } \\
\text { Forêt } 2016\end{array}$ & 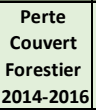 & $\begin{array}{l}\text { Couvert } \\
\text { Forêt } 2014\end{array}$ & $\begin{array}{l}\text { Couvert } \\
\text { Forêt } 2016\end{array}$ & \begin{tabular}{|c|} 
Perte \\
Couvert \\
Forestier \\
2014-2016 \\
\end{tabular} \\
\hline $\begin{array}{c}\text { Estimation } \\
\text { directe }\end{array}$ & 23099720 & 23063305 & 53100 & 23396020 & 23375600 & 40840 \\
\hline $\begin{array}{l}95 \% \text { Intervalle } \\
\text { de Confiance }\end{array}$ & \pm 455365 & \pm 456693 & \pm 20705 & \pm 647236 & \pm 648169 & \pm 56021 \\
\hline $\begin{array}{l}\text { Estimation } \\
\text { Carte }\end{array}$ & 23941404 & 23920637 & 20767 & 23941404 & 23920637 & 20767 \\
\hline $\begin{array}{l}\text { Estimation de } \\
\text { Régression }\end{array}$ & 23271194 & 23227807 & 60072 & 23191248 & 23170509 & 41159 \\
\hline $\begin{array}{l}95 \% \text { Intervalle } \\
\text { Confiance }\end{array}$ & \pm 220242 & \pm 221876 & \pm 20924 & \pm 461313 & \pm 461313 & \pm 40023 \\
\hline
\end{tabular}
perte à 321853 ha \pm 85800 ha. L'utilisation de la carte pour l'estimateur de régression améliore légèrement la précision des résultats.

\subsection{Estimations 2014-2016}

Tableau 7 : Estimations 2014-2016 selon les échantillons surfaciques et ponctuels.

Selon l'estimateur direct, la superficie du couvert forestier du Congo en 2016 est de l'ordre de 23,06 $\pm 0,46$ millions d'ha contre 23,23 $\pm 0,22$ millions d'ha selon l'estimateur de régression. L'estimateur le plus précis est celui obtenu via le modèle de régression car son intervalle de confiance est nettement plus faible.

Concernant les pertes du couvert forestier entre 2014 et 2016, l'estimateur direct présente une perte de 53000 ha \pm 20700 ha alors que l'estimateur de régression donne 60000 ha \pm 20900 ha de perte. Les intervalles de confiance de ces deux approches sont relativement identiques ce qui permet de conclure que l'utilisation de la carte n'apporte pas une amélioration significative de la précision dans ce cas de figure.

La perte du couvert forestier est de l'ordre de 17000 à 29000 ha/an entre 2000 et 2014 et semble avoir augmenté entre 2014 et 2016 : de l'ordre de 20000 à 40000 ha/an. 


\subsection{Synthèse graphique des estimations $\mathbf{2 0 0 0 - 2 0 1 4}$ et 2014-2016}

Les figures 10 et 11 permettent d'interpréter de manière synthétique les superficies et l'évolution du couvert forestier et surtout d'apprécier l'intervalle de confiance pour chacune des deux approches, celle par point et celle par analyse surfacique.

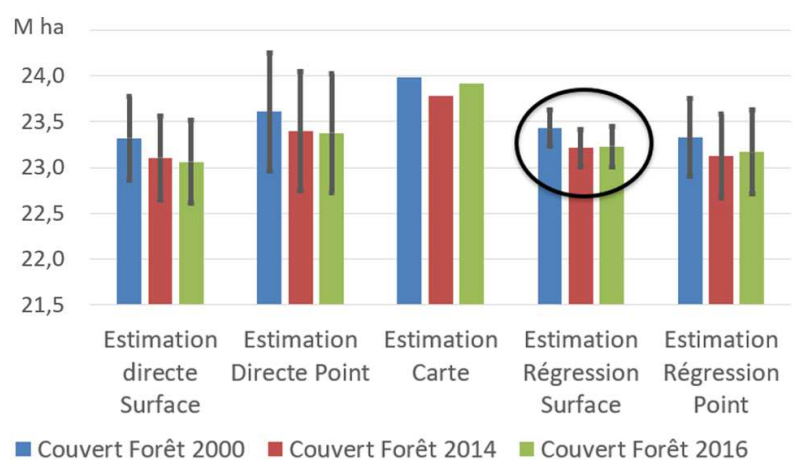

Figure 10 : Superficie du couvert forestier du Congo et ses intervalles de confiance.

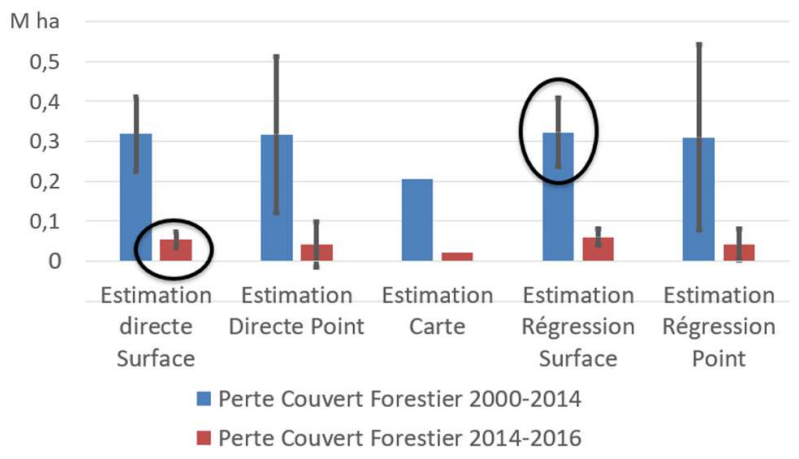

Figure 11 : Evolution du couvert forestier du Congo et ses intervalles de confiance.

Dans tous les cas évalués que ce soit pour la superficie totale du couvert forestier, de ses évolutions quelle que soit la période (2000-2014, 2014-2016) et la méthode prise en compte (directe ou régression), l'analyse surfacique présente une précision supérieure à l'analyse par point.

\section{Discussion}

Cette étude sur les estimations des superficies forestières et des pertes du couvert forestier de la République du Congo a révélé des écarts importants en comparant la méthode ponctuelle à la méthode surfacique. Les estimations les plus précises sont obtenues avec l'estimateur de régression. Cette amélioration de la précision relative est importante pour les superficies du couvert forestier mais elle est moindre voire inexistante pour les changements. Par ailleurs l'analyse surfacique apporte une précision supérieure à l'analyse par point quelles que soient la méthode ou la période considérée.

Les résultats statistiques des produits cartographiques bruts utilisés dans le cadre du processus REDD+ en République du Congo indiquent d'une manière générale une surestimation des superficies forestières d'une part et d'autre part une sous-estimation des pertes forestières. Ce constat semble être général à travers le monde à la vue des différentes études menées dans ce domaine tel que décrit par Bouetou Kadilamio et al. 2019 et Ngangoum Nana et al. 2018. Or tout changement sur les estimations des données d'activités a des conséquences sur les émissions des gaz à effet de serre mais aussi sur le niveau de référence des émissions définies par un pays. Ce qui est le cas de la République du Congo.

Dans le contexte de la REDD+ où il y a obligation pour les pays de bien évaluer et reporter les émissions des GES issues de la déforestation et de la dégradation des forêts, les estimations doivent être les plus proches possibles de la réalité terrain, et leurs incertitudes connues. La superficie du couvert forestier du Congo estimée dans le cadre de notre étude est de l'ordre de 23,2 $\pm 0,2$ millions ha en 2016. Cette statistique est largement en deçà des dernières estimations de la superficie forestière pour la République du Congo (24 millions d'ha, rapport troisième communication nationale). En effet le manque de continuité dans les chiffres sur les statistiques est une source d'interrogation sur la situation réelle de la forêt congolaise.

Selon l'estimateur de régression du tableau 6 , la perte du couvert forestier est de l'ordre de 17000 à 29000 ha/an entre 2000 et 2014 (avec une moyenne de 23000 ha/an \pm 6000 ha/an) et semble avoir augmenté entre 2014 et 2016 suivant le tableau 7 , entre 20000 et 40000 ha/an (avec une moyenne de 30000 ha/an \pm 10000 ha/an). $\mathrm{Si}$ ces résultats sont confirmés, cela aura des répercussions importantes sur la question de la durabilité des forêts de la république du Congo, mais aussi devrait conduire le pays à réécrire son NERF qui a été soumis en 2016 et qui affirmait que « le Congo est un pays à forte couverture forestière $(23,5$ millions d'hectares de forêts, représentant $69 \%$ du territoire national) et faible taux de déforestation et de dégradation forestière avec seulement $0,05 \%$ par an, soit environ 12000 hectares/an (NERF 2016). En effet des taux plus élevés des pertes de forêts suggèrent une plus grande perte des forêts congolaises et donc une perte plus importante de la biomasse mais aussi de la biodiversité végétale dans les forêts perdues (Tchatchou et al. 2015, Bouetou Kadilamio et al. 2019).

La présente étude n'a pas permis de déterminer avec exactitude les principaux moteurs de la déforestation et/ou de la dégradation forestière, mais le fait que les études passées aient faiblement cartographié les pertes suggère soit des activités inférieures à l'unité minimum de cartographie, soit des omissions lors de la photointerprétation des échantillons d'entrainement. En effet, la situation du couvert forestier, qui n'est pas uniforme sur l'ensemble du pays, varie en fonction de la densité de la population, de la qualité des infrastructures de transport, de la richesse des forêts, de l'historique de leur exploitation et de l'existence de zones urbaines (NERF, 2016). Aussi, plusieurs études ont mis en avant l'impact de la couverture nuageuse dans la cartographie des pertes forestières en utilisant les images optiques.

La méthode développée par Sannier et al, 2014, répond à ces exigences en privilégiant l'échantillonnage surfacique. Celui-ci permet d'obtenir des observations continues sur 
l'ensemble des valeurs de 0 à $100 \%$ de couvert forestier et donc par nature plus précises qu'un échantillonnage par point pour lesquels les observations sont binaires. Par ailleurs, cela permet aussi de réduire le biais, que l'on pourrait retrouver dans l'échantillonnage ponctuel, lié à la position des points situés à une interface forêt / non-forêt.

Finalement cette approche basée sur un échantillonnage surfacique est certes un peu plus complexe à mettre en œuvre, mais elle contribue à réduire considérablement le niveau d'incertitude. Elle est parfaitement extrapolable sur d'autres massifs forestiers et permet à la fois d'évaluer la précision de données cartographiques forestières ou thématiques existantes mais aussi de renforcer directement les estimations de surface du couvert forestier et de ses changements.

\section{Conclusion}

Au terme de cette étude qui est la première réalisée en République du Congo, plusieurs enseignements peuvent être tirés.

Les estimations les plus précises sont obtenues avec l'analyse surfacique et l'estimateur de régression, même si l'amélioration en comparaison avec la méthode directe est moindre voire inexistante pour les changements. Par ailleurs, l'estimateur de régression a plus d'intérêt lorsqu'il s'agit de données continues (surfaciques) que discrètes (ponctuelles). II n'a pas été possible d'évaluer le coût additionnel de l'interprétation des échantillons surfaciques par rapport aux échantillons ponctuels même si celui-ci semble relativement modeste dans le contexte de cette étude. II serait intéressant de réaliser une étude complémentaire afin d'évaluer si la réduction de l'intervalle de confiance de l'approche surfacique est économiquement bénéfique par rapport à une approche ponctuelle en prenant en compte l'effort additionnel d'interprétation des échantillons.

La précision relative est moindre pour les changements que pour la superficie totale du couvert forestier.

Dans l'optique d'améliorer la détection des changements par télédétection, l'utilisation de l'imagerie haute résolution comme Sentinel-2 $(10 \mathrm{~m})$ pourrait apporter un peu plus de précision pour les prochaines productions cartographiques.

Un autre enseignement important pour le Congo va être de renforcer le contrôle qualité et l'assurance qualité dans le processus d'élaboration des prochaines cartes de changement et d'occupation du sol. C'est du reste ce qui est envisagé dans le cadre de la révision du NERF de la République du Congo (lettre d'Intention CAFI, 2019). En perspectives, il serait bien de combiner l'utilisation des images optiques et des images radar afin d'améliorer la cartographie des pertes forestières, mais aussi l'estimation de la superficie de la couverture forestière.

\section{Remerciements}

Les auteurs remercient l'Agence Française de Développement (AFD) qui a financé cette étude très importante pour la République du Congo, ainsi que le projet OSFACO pour la sélection de ce projet.

Un remerciement particulier au CNIAF qui a mis à disposition de l'équipe toutes les données exogènes qui ont servi à la réalisation de cette étude.

Les auteurs remercient tout particulièrement la coordination nationale REDD+, l'université Marien N'GOUABI pour avoir facilité les formalités administratives tout au long de cette étude. Nous remercions également les sociétés IGN FI ainsi que SIRS-CLS pour leur soutien technique.

\section{Références}

Bouetou-Kadilamio L. N. O, Binsangou Malonga S, IFO S. A and Loumeto J. J, 2020. Field Validation of Land Cover and above Ground Carbon Mapping Using the Landsat OLI in Tropical Region. Research Journal of Environmental Sciences, 14: 18-29.

CNIAF, 2015. Cartographie du couvert forestier et des pertes de 2000 à 2014 de la république du Congo. Rapport. Brazzaville, Congo.

Congalton, R. G. (1991). A review of assessing the accuracy of classifications of remotely sensed data. Remote sensing of environment, 37(1), 35-46.

Coordination Nationale REDD, Jan 2016. Niveau des Emissions de Référence pour les Forêts (NERF) de la République du Congo, Soumission au Secrétariat CCNUCC,.65p.

Forêts d'Afrique Centrale Evaluées par Télédétection (FACET), 2012. Étendue et perte du couvert forestier en République du Congo de 2000 à 2010. Observatoire Satellital des Forêts d'Afrique Centrale (OSFAC). 110p. http://osfac.net/component/k2/itemlist/category/184-facet

Gallego, F. J. (1995). Sampling frames of square segments. Report EUR 16317 Luxembourg: European Commission.

GFOI (2013) - Intégration des données de télédétection et d'observation au sol pour l'estimation des émissions et des absorptions de gaz à effet de serre dans les forêts : Méthodes et pratiques recommandées par Initiative mondiale pour l'observation des forêts. Éditeur : Groupe sur l'observation de la Terre, Genève (Suisse), 2014. ISBN 978-92-990047-4-6

Lambin EF., and al, 2003. Dynamics of Land use and land cover change in Tropical region. Annu. Rev.Environ Resour. doi: 10.1146/ annu.rev. energy, pp206-231.

Matthew C Hansen, Alexander Krylov, Alexandra Tyukavina, Peter V Potapov, Svetlana Turubanova, Bryan Zutta, Suspense Ifo, Belinda Margono, Fred Stolle, Rebecca Moore, 2016: Humid tropical forest disturbance alerts using Landsat data. Environmental Research Letters 03/2016; 11(3): 034008. 
Ngangoum Nana T, Sannier C, Fichet L-V, Ghomsi H, Jaffrain $G$. Estimation par régression de l'incertitude des cartes de pertes de couvert forestier au Cameroun. Conférence OSFACO : Des images satellites pour la gestion durable des territoires en Afrique, Mar 2019, Cotonou, Bénin. ffhal-02189475f.

Olofsson P, Foody G.M., Stehman S.V., Woodcock C. E. Making better use of accuracy data in land change studies: Estimating accuracy and area and quantifying uncertainty using stratified estimation. Remote Sensing of Environment 129, 122-131(2013).

Olofsson P., Foody G.M., Herold M., Stehman S.V., Woodcock C.E., Wulder M.A. Good practices for estimating area and assessing accuracy of land change. Remote Sensing of Environment 148, $42-57$ (2014).

Ouissika B. C, Milandou C. S (2019). Cartographie du couvert forestier et des changements en République du Congo. Conférence OSFACO : Des images satellites pour la gestion durable des territoires en Afrique, Mar 2019, Cotonou, Bénin. ffhal-02189549f.

Parlement du Congo, (2020) Loi 33-2020, portant code forestier, Juillet 2020, Brazzaville Congo.

Présidence de la République (2019) : Lettre d'Intention portant sur l'établissement d'un partenariat de long terme visant la réalisation du Plan d'Investissement de la Stratégie Nationale REDD+ dans le cadre de l'Initiative pour la Forêt de l'Afrique Centrale (CAFI), Septembre 2019, Paris, France

Sannier C, McRoberts R A, Fichet LV and Massard (2014) Using the regression estimator with Landsat data to estimate proportion forest cover and net proportion deforestation in Gabon. ForestSat 2012 Special Issue. Remote Sensing of Environment, in press. http://dx.doi.org/10.1016/j.rse.2013.09.015.

Tchatchou B., Sonwa D. J., Ifo S. A., Tiana A. M., (2015). Déforestation et dégradation des forêts dans le Bassin du Congo : Etat des lieux, causes actuelles et perspectives. Papier occasionnel 120, Bogor, Indonésie, CIFOR.

Tyukavina, M. C. Hansen, P. Potapov, D. Parker, C. Okpa, S. V. Stehman, I. Kommareddy, S. Turubanova, 2018. Congo Basin forest loss dominated by increasing smallholder clearing. Sci. Adv. 4, eaat2993.

World Resources Institute (2017). Concessions forestières et aires protégées en République du Congo.
Titre en anglais:

\section{ANALYSIS AND CONSOLIDATION OF THE RESULTS ON ESTIMATES OF AREA OF FOREST COVER AND ITS CHANGES BETWEEN 2000 AND 2016 IN THE REPUBLIC OF CONGO}

\section{Légendes figures en anglais :}

Figure 1: Location map of the Republic of Congo (in red), in Central Africa.

Figure 2a: Plant diversity. Congo, Cuvette-Ouest Department (photo: Paul Godard)

Figure $\mathbf{2 b}$ : View of a mature forest in the north of the Republic of Congo in the Pokola FMU ${ }^{3}$. (Photo: Suspense Ifo, 2019)

Figure 3a: Logging in northern Republic of Congo in the Pokola FMU. (Photo: Suspense Ifo, 2019)

Figure 3b: Gigantism of tropical forest trees: a felled tree (Ayous, or Triplochiton scleroxylon) in the Pokola FMU (Photo: Suspense Ifo, 2018).

Figure 3c: View of a plot after logging in the Mpoukouogooué concession, Chaillu massif. (Photo: Suspense Ifo, 2018)

Figure 3d: Agricultural activity within the dense tropical forest of northern Congo. (Photo: Suspense Ifo, 2019)

Figure 4: Forestry map 2016 of the Republic of Congo

Figure 5: Overview of satellite image coverage of the 3 pivot dates.

Figure 6: Main steps of stratification

Figure 7: Overview of the final distribution of samples by strata across the Congolese territory

Figure 8: Mapping method by photo-interpretation of samples

Figure 9: Mapping of forest cover changes between 2000, 1014 and 2016 on a sample (Near Mboko town)

Figure 10: Congo's forest cover area and its confidence intervals.

Figure 11: Congo's forest cover change and its confidence intervals

${ }^{3}$ Forest Management Unit 